\title{
Performance of A Sodium Thiocyanate Based Gel Polymer Electrolyte In Redox Capacitors
}

\section{C.M. Bandaranayake, Y.M.C.D. Jayathilake, K.P. Vidanapathirana and K.S. Perera}

Polymer Electronics Research Laboratory, Department of Electronics, Faculty of Applied Sciences, Wayamba University of Sri Lanka, Kuliyapitiya, Sri Lanka.kumudu31966@gmail.com

\begin{abstract}
Solid state redox capacitors have received a tremendous interest in terms of several characteristics such as their fast energy delivery, short charging time, high power density and extended durability. Due to the absence of a liquid electrolyte, they are free from drawbacks of leakage and unsafe. In this study, preparation and characterization of a redox capacitor consisting with two identical polypyrrole (PPy): dodecylbenzenesulfonate (DBS) electrodes and a gel polymer electrolyte (GPE) based on polyvinylidene fluoride (PVdF), ethylene carbonate (EC), propylene carbonate (PC) and sodium thiocyanate (NaSCN) is reported. The GPE having the composition, 0.4 PVdF: 1 EC: 1 PC: $0.075 \mathrm{NaSCN}$ (by weight) showed the maximum conductivity of $2.25 \times 10^{-3} \mathrm{~S} \mathrm{~cm}^{-1}$. Redox capacitors were characterized using Cyclic Voltammetry, Electrochemical Impedance Spectroscopy and Galvanostatic Charge Discharge test. They exhibited an energy density about $0.25 \mathrm{~W} \mathrm{~h} \mathrm{~kg}^{-1}$ and an average power density of $4 \mathrm{~kW} \mathrm{~kg}^{-1}$
\end{abstract}

Keywords: gel polymer electrolytes, conducting polymers, impedance spectroscopy, ionic conductivity, redox capacitors

\section{Introduction}

Introducing a more sustainable energy economy has been identified as a necessity of the era due to the growing concern about the fossil fuel crisis. Supercapacitors arouse wide range of research activities as a type of novel electrochemical energy storage devices because of their attractive properties such as fast and high energy delivery, short charging time, high power density, extended durability and environmental friendliness (Yu et al., 2012; Zhu et al., 2011). Due to those properties they have the capability to meet requirements of many modern applications such as hybrid vehicles, mobile phones, computers, automatic door closers, medical equipment and for load leveling (Hashmi et al., 2005; Ma et al., 2015) There are two types of supercapacitors namely, electrochemical double layer capacitors (EDLCs) and redox/ pseudo capacitors. Difference between these two types is the energy storage 
mechanism. In EDLCs, capacitance arises by building up of charges at the electrode/electrolyte interface and various forms of carbon based materials are used as electrodes. But in redox capacitors, charges are stored in bulk of a material in response to redox reactions. Electrode materials such as $\mathrm{RuO}_{2}$, $\mathrm{MnO}_{2}, \mathrm{CoO}_{\mathrm{x}}$ and most importantly conducting polymers are employed for redox capacitors (Snook et al., 2011; Hashmi et al., 2005; Gupta \& Tripathi 2013) Conducting polymers have been identified as one of the most suitable electrode material in redox capacitors due to their fast doping and dedoping ability, high charge density, easy synthesis and low cost.

Electrolyte plays a great role during charge discharge process in determining the capacity. Therefore, an electrolyte with good electrochemical properties is very important for enhancing efficiency (Ma et al., 2015). Number of redox capacitors based on liquid electrolytes can be found in literature, but those are associated with usual disadvantages such as corrosion, self discharge, low energy density, bulky design, leakage etc. (Hashmi et al., 1998; Hashmi et al., 2005) These issues can be skirted using gel polymer electrolytes (GPEs) which have been considered to use for various applications due to their merits of high ionic conductivity and good mechanical properties (Perera et al., 2007, Jayathilake et al., 2014). Therefore, development of solid state redox capacitors using GPEs is one of the current research areas of great interest (Hashmi et al., 2005). GPEs are prepared by immobilizing a salt solvent mixture in a suitable polymer matrix. Due to this special structure, they give rise to liquid like conductivity and preferable mechanical stability like solid electrolytes. GPEs are ideal candidates for redox capacitors due to excellent chemical stability, mechanical properties etc. Up to date there are very few reports about GPE based redox capacitors

In this paper, it is reported about a redox capacitor fabricated with polypyrrole (PPy): dodecylbenzenesulfonate (DBS) electrodes and a GPE based on polyvinylidene fluoride $(\mathrm{PVdF})$, ethylene carbonate (EC), propylene carbonate (PC) and sodium thiocyanate $(\mathrm{NaSCN})$. GPE was characterized by Electrochemical Impedance Spectroscopy and DC Polarization test. The performance of redox capacitors have been evaluated by Electrochemical Impedance Spectroscopy (EIS), Cyclic Voltammetry (CV) and Galvanostatic Charge Discharge (GCD) test.

\section{Methodology}

\section{Preparation of gel polymer electrolyte and redox capacitors}

Polyvinylidene fluoride (PVdF) (534,000 MW), ethylene carbonate (EC) $(98 \%)$, propylene carbonate (PC) $(99 \%)$ and sodium thiocyanate (NaSCN) $(98 \%)$ were purchased from Aldrich and used without further purification. For 
the preparation of GPE, 0.5 PVdF: 1.0 EC: 1.0 PC: 0.1 NaSCN (weight basis) was selected as the initial composition. First, required amount of $\mathrm{NaSCN}$ was dissolved in EC and PC by magnetic stirring. Then, PVdF was added in to the mixture and heating was done at $120^{\circ} \mathrm{C}$ for about 30 minutes. Finally the resultant hot mixture was pressed in between two well cleaned glass plates. Number of samples were prepared by changing the salt and the polymer concentration to find the optimum composition that results the highest ionic conductivity. EC and PC weight ratio was fixed as 1:1 throughout the study.

PPy: DBS electrodes were prepared using 0.1 M pyrrole (98\%, Aldrich) and $0.05 \mathrm{M}$ sodium dodecylbenzenesulfonate (SDBS) (Aldrich). Films of area, $1 \times 1$ $\mathrm{cm}^{2}$ were electrochemically polymerized on fluorine-doped tin oxide (FTO) glass plates using a computer controlled three electrode set up. A standard Ag/ $\mathrm{AgCl}_{2}$ electrode was used as the reference electrode and a Pt electrode was used the counter electrode. The highest conducting GPE film of $1 \times 1 \mathrm{~cm}^{2}$ was sandwiched in between two PPy: DBS electrodes to fabricate redox capacitors in the configuration of PPy: DBS / PVdF: EC: PC: NaSCN / PPy: DBS.

\section{Electrochemical measurements}

A circular shaped pellet of GPE was sandwiched in between two stainless steel (SS) electrodes. Impedance data were collected in the frequency range from $0.4 \mathrm{MHz}$ to $10.0 \mathrm{mHz}$ using a computer controlled Metrohm Autolab M101 impedance analyzer. Measurements were taken from $30{ }^{\circ} \mathrm{C}$ to $55^{\circ} \mathrm{C}$ using a glass tube furnace in order to trace the composition that results the highest ionic conductivity and to study the conductivity variation with temperature. The transference number measurements of the composition that exhibited the highest conductivity were done by DC polarization test. A circular film of GPE was sandwiched in between two SS blocking electrodes and current variation through the cell was measured as a function of time under $1.0 \mathrm{~V} \mathrm{DC}$ bias potential.

EIS measurements of redox capacitors were taken using a computer controlled Metrohm Autolab M101 impedance analyzer in the frequency range of 0.4 $\mathrm{MHz}$ to $10 \mathrm{mHz}$. Overall specific capacitance of the capacitors at the lowest frequency was evaluated by using the equation 1 (Tey et al., 2016).

$$
\mathrm{C}=-\frac{1}{\omega \mathrm{Z}^{\prime \prime}}
$$

where, $\omega(=2 \pi f)$ is angular frequency and $Z$ " is the imaginary part of the impedance at a low frequency value.

$\mathrm{CV}$ tests were done at the scan rate of $10 \mathrm{mVs}^{-1}$ in order to find out the most suitable potential window for redox capacitors. The potential window was gradually increased from $0.8 \mathrm{~V}$ to $2.0 \mathrm{~V}$. For the potential window which gave 
the highest capacity, CV tests were done by changing scan rates. Capacitance was evaluated using the equation (2) where $\int \mathrm{I}(\mathrm{V}) \mathrm{dV}$ is total integral area of the cyclic voltammogram, $\mathrm{s}$ is the scan rate and $\Delta \mathrm{V}$ is the width of the potential window (Beidaghi \& Wang 2011; Tey et al., 2016).

$$
C=\frac{\int I(V) d V}{s \Delta V}
$$

Galvanostatic Charge discharge tests were done at the constant current of 5 $\mathrm{mA}$. Redox capacitor was first discharged up to $0.0 \mathrm{~V}$ and immediately charged up to $1.8 \mathrm{~V}$. Using the equation (3) and the linear part of the discharge curve, the discharge capacitance was calculated (Tripathi et al., 2013).

$$
\mathrm{C}=\frac{\mathrm{i} \Delta \mathrm{t}}{\Delta \mathrm{V}}
$$

where $\mathrm{i}$ is the discharge current, $\Delta \mathrm{t}$ is the time interval for the change of voltage $\Delta \mathrm{V}$.

The energy density (E) and power density (P) were calculated by using below equations.

$$
\begin{aligned}
& \mathrm{E}=\frac{1}{2} \mathrm{C}(\Delta \mathrm{V})^{2} \\
& \mathrm{P}=\frac{\mathrm{E}}{\mathrm{t}}
\end{aligned}
$$

where $\mathrm{C}$ is the capacitance, is the potential difference and $\mathrm{t}$ is the total discharge time.

\section{Results and Discussion}

A resultant Nyquist plot of SS / PVdF: EC: PC: NaSCN / SS symmetric cell is shown in figure 01 . It exhibits only a part of a semicircle. For a symmetric cell with two blocking electrodes, theoretically there should be two semicircles and a spike (Jayathilake et al., 2014). One semicircle is in the intermediate frequency region and the other one is in the high frequency region. The spike appears in the low frequency region. First semicircle represents the bulk electrolyte properties while the second one represents the interfacial properties. Inset of figure 01 shows the high frequency region of the resultant Nyquist plot. It is seen that the high frequency semicircle is not present. Unavailability of required high frequency might be the possible reason for this observation (Jayathilake et al., 2015). The spike is also absent in the resultant Nyquist plot and it may be also due to unavailability of required low frequency range. Bulk electrolyte resistance, $\mathrm{R}_{\mathrm{b}}$ was evaluated from the resultant impedance plot using nonlinear least square fitting program (Jayathilake et al., 2014). The equivalent circuit used is given in figure 02 . Conductivity of the electrolyte 
was calculated using the $R_{b}$ value and equation (6) where $t$ is thickness and $A$ is area of the GPE film.

$$
\sigma=\frac{\mathrm{t}}{\mathrm{R}_{\mathrm{b}} \mathrm{A}}
$$

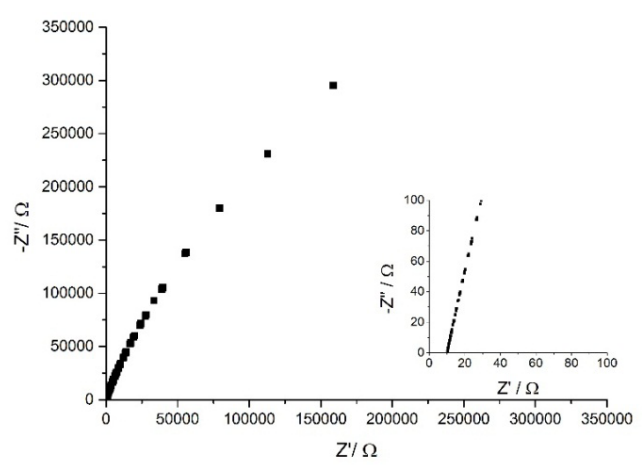

Figure 01: A resulted Nyquist plot for the SS / PVdF: EC: PC: NaSCN / SS cell where Z' and Z" are real and imaginary parts of the impedance. High frequency part of the plot has been shown in the inset.

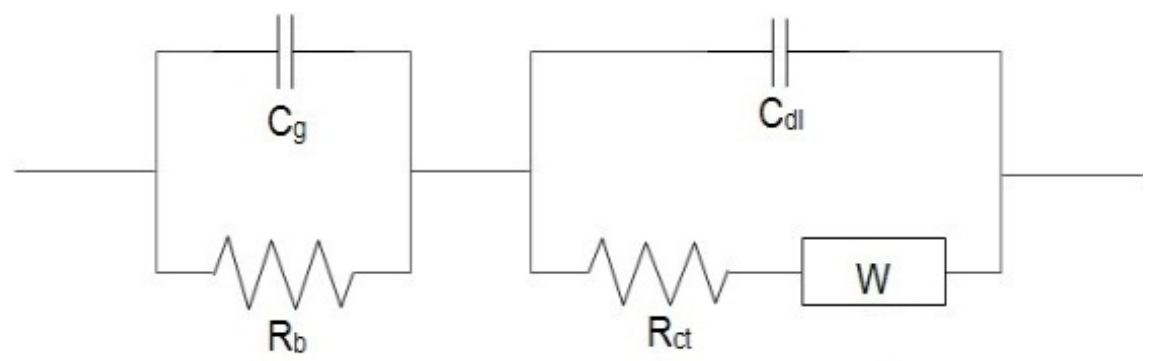

Figure 02 The equivalent circuit for SS / GPE / SS cell. where $\mathrm{R}_{\mathrm{b}}$ - bulk electrolyte resistance, $\mathrm{R}_{\mathrm{ct}}$ - interfacial resistance, $\mathrm{C}_{\mathrm{g}}$ - geometrical capacitance, $\mathrm{C}_{\mathrm{dl}}$ interfacial capacitance and $\mathrm{W}$ - Warburg impedance.

Room temperature conductivity variation of GPE with $\mathrm{NaSCN}$ concentration (by weight) is shown in figure 03. Conductivity has increased while increasing the weight ratio of salt up to 0.075 and thereafter it has decreased. When increasing the NaSCN concentration, number of charge carriers is also increasing and due to that, ionic conductivity of GPE is also increasing. Further increment of salt can lead to create ion aggregates in GPE so that number of free ions which participate for conductivity also decreases. Thus, the conductivity decreases (Osman et al., 2012). The highest conducting sample (PVdF 0.5: EC 1.0: PC 1.0: NaSCN 0.075) was further optimized by changing the concentration of the polymer. 


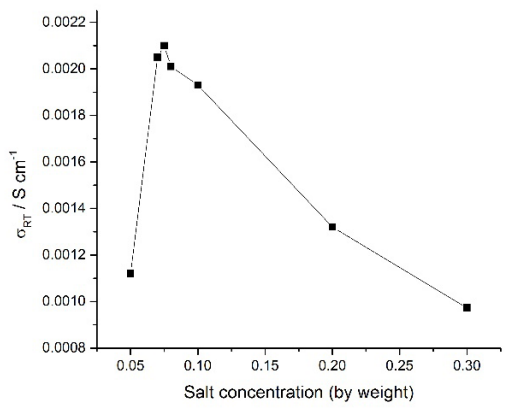

Figure 03 Variation of ionic conductivity at room temperature with NaSCN concentration (by weight)

Conductivity variation with the polymer concentration is shown in figure 04 .

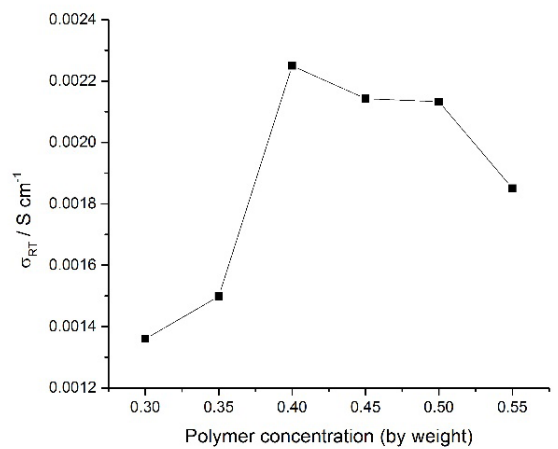

Figure 04: Variation of ionic conductivity at room temperature with PVDF concentration (by weight)

Initially the conductivity increases with polymer concentration and after 0.4, it decreases gradually. Increment of the conductivity with polymer concentration may be due to the increasing of the assistance for ionic motion by polymer chains. When increasing polymer concentration further, it increases the viscosity of the system (Sharma \& Sekhon 2006). Because of that, the resistance for ion mobility increases and the conductivity decreases. The sample having the composition $0.4 \mathrm{PVdF}: 1.0 \mathrm{EC}: 1.0 \mathrm{PC}: 0.075 \mathrm{NaSCN}$ was selected as the optimum composition because it showed the maximum ionic conductivity of $2.25 \times 10^{-3} \mathrm{~S} \mathrm{~cm}^{-1}$ at room temperature. Further, it was of free standing nature.

Figure 05 shows the $\ln (\sigma)$ variation as a function of $1000 /$ T. The plot shows a linear behavior implying that it follows Arrhenius behavior. That is the conductivity of the respective GPE takes place via the hopping mechanism. Ions of the GPE are supposed to jump in to the neighboring vacant site and cause 
the conductivity (Ramesh \& Chai 2007). Enhancing of the ionic conductivity with increasing temperature shows that the GPE is thermally active. When temperature of the GPE is increased, the ions gain more energy and as a result of that, ion mobility increases. This increases the ionic conductivity with temperature. The activation energy obtained using the slope of the plot is $0.16 \mathrm{eV}$. This value of activation energy is very much in agreement of previous reports (Osman et al., 2012). The low activation energy may be due to completely amorphous nature of the GPE.

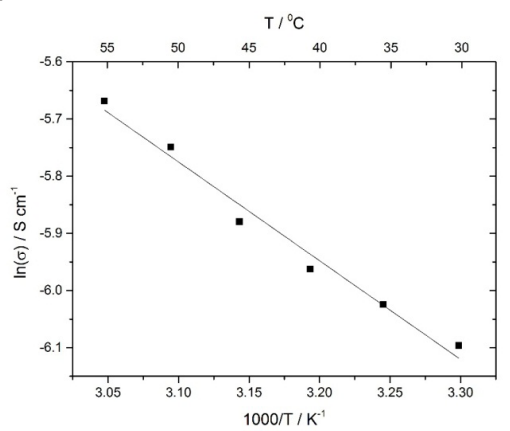

Figure 05: Variation of ionic conductivity of 0.4 PVdF: 1.0 EC: 1.0 PC: $0.075 \mathrm{NaSCN}$ GPE as a function of temperature

Figure 06 shows current variation across the GPE assembled between two SS electrodes as a function of time. A sudden drop of current can be seen during the first hour due to polarization of ions. After that, it tends to stabilize due to electron movement. Using the equation (7), the ionic transference number was calculated.

$$
t_{i}=\frac{i_{t}-i_{e}}{i_{t}}
$$

where $i_{t}$ is the total current due to ions and electrons and $i_{e}$ is the current due to electrons (Jayathilake et al., 2015). Calculated ionic transference number was 0.94. This indicates that the GPE is predominantly a good ionic conductor. Value of ionic transference number obtained in this study is nearly equal to the values reported by Chandra et al., and Kumar et al., for sodium ion conducting polymer electrolytes (Chandra et al., 2012; Kumar \& Hashmi 2010).

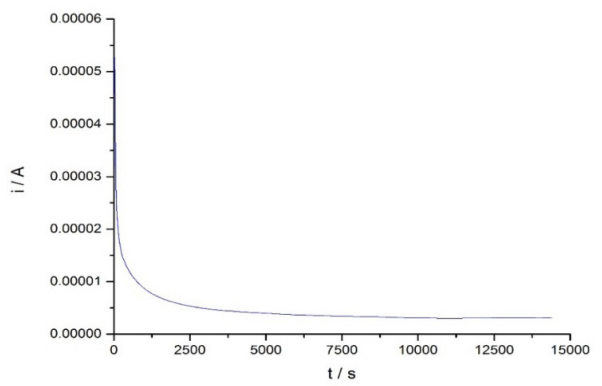

Figure 06: Variation of the current through SS / 0.4 PVdF: 1.0 EC: 1.0 PC: 0.075 $\mathrm{NaSCN} / \mathrm{SS}$ cell with time under $1.0 \mathrm{~V}$ bias voltage. 
A resultant impedance plot of redox capacitor is shown in figure 07 . There is a semicircle in the middle frequency region and two linear regions at low frequencies. The latter features indicate that the capacitive behavior is existing at low frequencies (Jain \& Tripathi 2013) based on the double-layer capacitance of high specific surface area carbon materials, are attracting major fundamental and technological interest as highly reversible, electrical-charge storage and delivery devices, capable of being operated at high power densities. In the present paper, studies have been carried out on nanocomposite gel polymer electrolyte comprising poly(vinylidene fluoride-co-hexafluoropropylene. The mid frequency semicircle represents charge transfer process at electrode/ electrolyte interface. The first intercept of the semicircle with the real axis is assigned to bulk electrolyte resistance. (Girija \& Sangaranarayanan 2006). The first linear region represents the capacitive behavior due to Warburg diffusion of ions (Zhu et al., 2011). The next linear region which is very steep resembles the capacitive response of an ideal capacitor. Bulk electrolyte resistance and charge transfer resistance evaluated from nonlinear least square fitting program are respectively $44.9 \Omega$ and $549.6 \Omega$. The calculated specific capacitance value at $10 \mathrm{mHz}$ was $12 \mathrm{mF} \mathrm{cm}^{-2}$ or an equivalent of $48 \mathrm{~F} \mathrm{~g}^{-1}$.

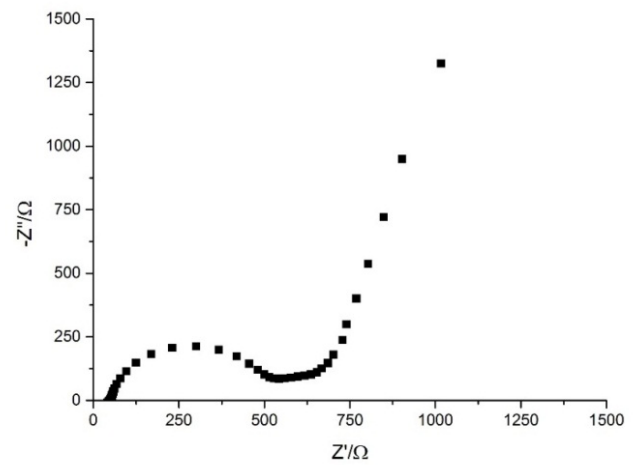

Figure 07: Impedance plot for PPy: DBS / PVdF: EC: PC: NaSCN / PPy: DBS redox capacitor.

Cyclic voltammograms of PPy: DBS / PVdF: EC: PC: NaSCN / PPy: DBS redox capacitors in different potential windows are shown in figure 08 .

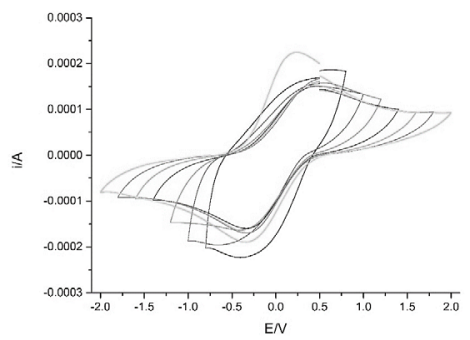

Figure 08: Cyclic voltammograms for the PPy: DBS / PVdF: EC: PC: NaSCN / PPy: DBS redox capacitor in different potential windows. 
A nearly rectangular shape cyclic voltammogram can be seen in $-0.8 \mathrm{~V}$ to 0.8 $\mathrm{V}$ potential window and while increasing the potential window, two symmetric anodic and cathodic peaks appeared. In redox capacitors, charge storage is due to the redox reaction at electrode/electrolyte interface. The appearance of oxidation and reduction peaks reveals the contribution of redox process to the capacity (Yu et al., 2012) Absence of peaks in $-0.8 \mathrm{~V}$ to $0.8 \mathrm{~V}$ potential window may be due to the absence of redox reactions in this potential window. The potential window from $-1.8 \mathrm{~V}$ to $1.8 \mathrm{~V}$ was selected as the most suitable window.

Resultant cyclic voltammograms for the scan rates of 5, 10 and $15 \mathrm{mV} \mathrm{s}^{-1}$ are shown in figure 09. The response of each redox capacitor shows a dependency on scan rate. In cyclic voltammograms, a shift of peak positions can be seen with scan rates. Peak current of redox capacitors also increases with peak shifting. This is attributed to the pseudocapacitance of redox process at the electrode/electrolyte interface ( $\mathrm{Yu}$ et al., 2012) With increasing scan rate, capacitance increased possibly due to improved redox reactions. The scan rate of $10 \mathrm{mV} \mathrm{s}^{-1}$ gives the highest capacity of $32 \mathrm{mF} \mathrm{cm}^{-2}\left(129.6 \mathrm{~F} \mathrm{~g}^{-1}\right)$. After this scan rate, capacity decreased when the scan rate was increased. This may be due to occurrence of incomplete redox reactions which do not allow full charge storage. (Wang et al., 2013; Fonseca et al., 2006).

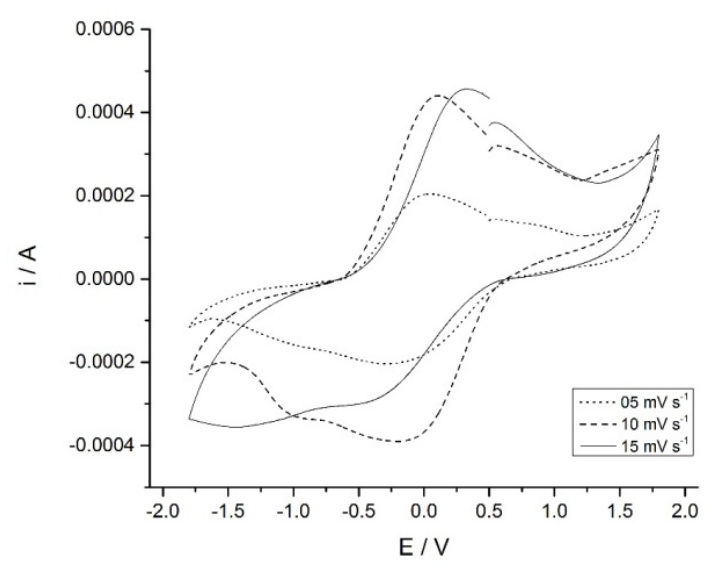

Figure 09: Cyclic voltammograms for PPy: DBS / PVdF: EC: PC: NaSCN / PPy: DBS redox capacitor at different scan rates.

Galvanostatic charge discharge (GCD) characteristics of redox capacitor is shown in figure 10. The charge discharge curve shows nonlinear behavior. This non linear behavior may be due to the redox reaction at electrode/electrolyte interface (Hashmi \& Updahyaya 2002). Discharge capacity of redox capacitor was about $13.8 \mathrm{mF} \mathrm{cm}^{-2}$ or an equivalent of $55 \mathrm{~F} \mathrm{~g} \mathrm{~g}^{-1}$. Energy density was about $0.25 \mathrm{~W} \mathrm{~h} \mathrm{~kg}^{-1}$ and power density was about $4 \mathrm{~kW} \mathrm{~kg}^{-1}$. 


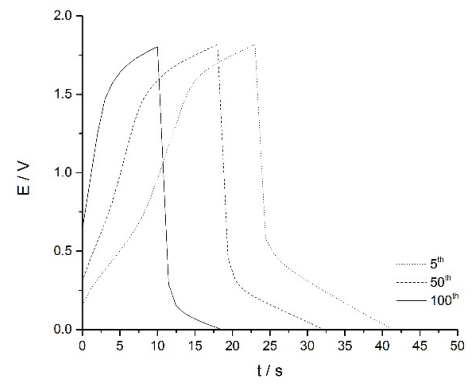

Figure 10: Charge discharge curve for PPy: DBS / PVdF: EC: PC: NaSCN / PPy: DBS redox capacitor at charge discharge current of $0.5 \mathrm{~mA}$.

\section{Conclusions}

The GPE prepared with 0.4 PVdF: 1.0 EC: 1.0 PC: $0.075 \mathrm{NaSCN}$ showed the maximum conductivity of $2.25 \times 10^{-3} \mathrm{~S} \mathrm{~cm}^{-1}$ and a freestanding nature. Conductivity variation with temperature followed Arrhenius behavior with the activation energy of $0.16 \mathrm{eV}$. That low activation energy supports the high conductivity of the GPE. Ionic transference number obtained from DC polarization test with blocking electrodes was 0.94. This indicates the GPE was purely an ionic conductor. Resultant impedance plot of PPy: DBS / 0.4 PVdF: 1.0 EC: 1.0 PC: 0.075 NaSCN / PPy: DBS redox capacitor shows capacitive nature at low frequencies. Calculated capacitance of the EDLC at $10 \mathrm{mHz}$ is $12 \mathrm{mF} \mathrm{cm}^{-2}$ (equivalent to a single electrode specific capacitance of $\left.24 \mathrm{~F} \mathrm{~g}^{-1}\right)$. Highest capacitance of $32.4 \mathrm{mF} \mathrm{cm}^{-2}\left(129.6 \mathrm{~F} \mathrm{~g}^{-1}\right)$ was obtained from the cyclic voltammetry test at the scan rate of $10 \mathrm{mV} \mathrm{s}^{-1}$. Continuous cycling of the redox capacitor showed that the capacity fade was slightly high. Results of the Galvanostatic Charge Discharge test showed that the discharge capacity was about $13.5 \mathrm{mF} \mathrm{cm}^{-2}\left(27 \mathrm{~F} \mathrm{~g}^{-1}\right)$. Further, energy density was found to be about $0.25 \mathrm{~W} \mathrm{~h} \mathrm{~kg}^{-1}$ whereas power density was about $4 \mathrm{~kW} \mathrm{~kg}^{-1}$.

\section{Acknowledgements}

Financial assistance from National Research Council of Sri Lanka (NRC 12109) and University Grants Commission (UGC/VC/DRIC/IRG-2014/WUSL) are highly acknowledged.

\section{References}

Beidaghi, M. \& Wang, C., (2011). Electrochimica Acta Micro-supercapacitors based on three dimensional interdigital polypyrrole / C-MEMS electrodes. Electrochimica Acta, 56(25), pp.9508-9514. Available at: http://dx.doi. org/10.1016/j.electacta.2011.08.054. 
Chandra, A., Chandra, A. \& Thakur, K., (2012). Na+ Ion Conducting Hot-pressed Nano Composite Polymer Electrolytes. Portugaliae Electrochimica Acta, 30(2), pp.81-88. DOI: http://dx.doi.org/10.4152/ pea.201202081

Fonseca, C.P., Benedetti, J.E. \& Neves, S., (2006). Poly(3-methyl thiophene)/ PVDF composite as an electrode for supercapacitors. Journal of Power Sources, 158(1), pp.789-794. DOI: http://dx.doi.org/10.1016/j. jpowsour.2005.08.050

Girija, T.C. \& Sangaranarayanan, M. V., (2006). Polyaniline-based nickel electrodes for electrochemical supercapacitors-Influence of Triton X-100. Journal of Power Sources, 159(2), pp.1519-1526. DOI: http:// dx.doi.org/10.1016/j.jpowsour.2005.11.078

Gupta, A. \& Tripathi, S.K., (2013). Effect of anionic size of PMMA Based Polymer Gel Electrolytes for Redox Capacitor. International Journal of Engineering Research and Applications, 3(1), pp.1908-1911.

Hashmi, S. \& Updahyaya, H., (2002). MnO2-polypyrrole conducting polymer composite electrodes for electrochemical redox supercapacitors. Ionics, 8, pp.272-277. Available at: http://link.springer.com/article/10.1007/ BF02376079. DOI: http://dx.doi.org/10.1007/bf02376079

Hashmi, S.A. et al., (1998). Conducting polymer-based electrochemical redox supercapacitors using proton and lithium ion conducting polymer electrolytes. Polymer International, 47(1), pp.28-33. DOI: http://dx.doi.org/10.1002/(sici)1097-0126(199809)47:1\%3C28::aidpi3\%3E3.0.co;2-c

Hashmi, S.A., Kumar, A. \& Tripathi, S.K., (2005). Investigations on electrochemical supercapacitors using polypyrrole redox electrodes and PMMA based gel electrolytes. European Polymer Journal, 41(6), pp.1373-1379. Available at: http://www.sciencedirect.com/science/ article/pii/S0014305705000091. DOI: http://dx.doi.org/10.1016/j. eurpolymj.2004.12.013

Jain, A. \& Tripathi, S.K., (2013). Experimental studies on high-performance supercapacitor based on nanogel polymer electrolyte with treated activated charcoal. Ionics, 19(3), pp.549-557. Available at: http://www. scopus.com/inward/record.url?eid=2-s2.0-84874311440\&partnerID $=40$ \&md5=340fad731cfad92e64f79dd626b12839.

Jayathilake, Y.M.C.D. et al., (2014). A novel gel polymer electrolyte based on Polymethylmethacrylate and Copper Trifluoromethanesulfonate. Journal of Electroanalytical Chemistry, 724, pp.125-129. Available at: http:// linkinghub.elsevier.com/retrieve/pii/S1572665714001726. DOI: http:// dx.doi.org/10.1016/j.jelechem.2014.04.018 
Jayathilake, Y.M.C.D., Perera, K.S. \& Vidanapathirana, K.P., (2015). Preparation and characterization of a polyacrylonitrile-based gel polymer electrolyte complexed with 1 methyl-3 propyl immidazolium iodide. Journal of Solid State Electrochemistry, 19, pp.2199-2203. Available at: http://link.springer.com/10.1007/s10008-015-2834-7. DOI: http://dx.doi. org/10.1007/s10008-015-2834-7

Kumar, D. \& Hashmi, S.A., (2010). Ionic liquid based sodium ion conducting gel polymer electrolytes. Solid State Ionics, 181(8-10), pp.416-423. Available at: http://dx.doi.org/10.1016/j.ssi.2010.01.025.

Ma, G. et al., (2015). A redox mediator doped gel polymer as an electrolyte and separator for a high performance solid state supercapacitor. Journal of Materials Chemistry A, 3(7), pp.4035-4041. Available at: http:// dx.doi.org/10.1039/C4TA06322H\nhttp://pubs.rsc.org/en/content/ articlepdf/2015/ta/c4ta06322h.

Osman, Z. et al., (2012). AC ionic conductivity and DC polarization method of lithium ion transport in PMMA - LiBF4 gel polymer electrolytes. Results in Physics, 2, pp.1-4. DOI: http://dx.doi.org/10.1016/j.rinp.2011.12.001

Perera, K., Vidanapathirana, K.P. \& Dissanayake, M.A.K.L., (2007). Artificial muscles based on Polyacrylonitrile based polymer electrolyte. Sri Lankan Journal of Physics, 8, pp.39-45. DOI: http://dx.doi.org/10.4038/sljp. v8i0.212

Ramesh, S. \& Chai, M.F., (2007). Conductivity, dielectric behavior and FTIR studies of high molecular weight poly(vinylchloride)-lithium triflate polymer electrolytes. Materials Science and Engineering: B, 139(23), pp.240-245. Available at: http://www.sciencedirect.com/science/ article/pii/S0921510707001316. DOI: http://dx.doi.org/10.1016/j. mseb.2007.03.003

Sharma, J.P. \& Sekhon, S.S., (2006). PMMA-based polymer gel electrolytes containing NH4PF6: Role of molecular weight of polymer. Materials Science and Engineering: B, 129(1-3), pp.104-108. Available at: http:// linkinghub.elsevier.com/retrieve/pii/S0921510706000043. DOI: http:// dx.doi.org/10.1016/j.mseb.2005.12.019

Snook, G.A., Kao, P. \& Best, A.S., (2011). Conducting-polymer-based supercapacitor devices and electrodes. Journal of Power Sources, 196(1), pp.1-12. Available at: http://dx.doi.org/10.1016/j.jpowsour.2010.06.084.

Tey, J.P. et al., (2016). Durian shell-based activated carbon electrode for EDLCs. Ionics. Available at: http://link.springer.com/10.1007/s11581016-1640-2. 
Tripathi, S.K. et al., (2013). Studies on redox supercapacitor using electrochemically synthesized polypyrrole as electrode material using blend polymer gel electrolyte. Indian Journal of Pure \& Applied Physics, 51(May), pp.315-319.

Wang, W. et al., (2013). Three dimensional few layer graphene and carbon nanotube foam architectures for high fidelity supercapacitors. Nano Energy, 2(2), pp.294-303. Available at: http://dx.doi.org/10.1016/j. nanoen.2012.10.001.

Yu, H. et al., (2012). A novel redox-mediated gel polymer electrolyte for highperformance supercapacitor. Journal of Power Sources, 198, pp.402-407. Available at: http://dx.doi.org/10.1016/j.jpowsour.2011.09.110.

Zhu, Y. et al., (2011). Carbon-based supercapacitors produced by activation of graphene. Science (New York, N.Y.), 332(6037), pp.1537-1541. DOI: http://dx.doi.org/10.1126/science. 1200770 